\title{
ON GENERATING DISTRIBUTIVE SUBLATTICES OF ORTHOMODULAR LATTICES
}

\author{
RICHARD J. GREECHIE
}

\begin{abstract}
A Foulis-Holland set is a nonempty subset $S$ of an orthomodular lattice such that whenever $x, y, z$ are distinct elements of $S$ one of them commutes with the other two. If $S$ is a Foulis-Holland set, then the sublattice generated by $S$ is distributive.
\end{abstract}

The purpose of this paper is to prove the following: If $S$ is a subset of an orthomodular lattice such that whenever $T$ is a three-element subset of $S$ one of the elements of $T$ commutes with the other two, then the sublattice generated by $S$ is distributive. This result extends the well-known FoulisHolland Theorem, the not-so-well-known Marsden-Herman Theorem, as well as a recent result of $\mathrm{G}$. Crown. The result appears to be new even for the orthomodular lattice of all (orthogonal) projections on a Hilbert space.

1. Preliminaries. Throughout this paper $L$ will denote an orthomodular lattice, that is, a bounded lattice $(L, \vee, \wedge, 0,1)$ together with an orthocomplementation ': $L \rightarrow L$ satisfying $x \vee x^{\prime}=1, x^{\prime \prime}=x, x^{\prime} \vee y^{\prime}=(x \wedge y)^{\prime}$ and $y=x \vee\left(y \wedge x^{\prime}\right)$ whenever $x \leqslant y$. Boolean algebras and the projection lattices of von Neumann algebras are the prototypical examples. Because of the latter class of examples some of the notation is derived from Hilbert space theory. For example, for $a, b \in L$, we write $a \perp b$ in case $a \leqslant b^{\prime}, a \oplus b=a$ $\vee b$ in case $a \perp b$, and $b-a=b \wedge a^{\prime}$ in case $a \leqslant b$; also we say that $a$ commutes with $b$, written $a \mathbf{C} b$, in case $b=(a \wedge b) \oplus\left(a^{\prime} \wedge b\right)$. It is a fact that $a \mathbf{C} b$ implies $b \mathbf{C} a$. For subsets $A$ and $B$ of $L$ we write $A \perp B$ (resp. $A \mathbf{C} B$ ) in case $a \perp b$ (resp., $a \mathbf{C} b$ ) for all $a \in A$ and $b \in B$. Any pair of elements commute in a Boolean algebra and two (orthogonal) projections on a Hilbert space commute in the above sense if and only if they commute in the usual algebraic sense. For any subset $M$ of $L$, the centralizer $C(M)$ of $M$, consisting of those elements of $L$ which commute with each element of $M$, is a subcomplete suborthomodular sublattice of $L$. In particular, if $a$ and $b$ are in $C(M)$ then so is any lattice polynomial in $a, a^{\prime}, b$ and $b^{\prime}$. The set $C(L)$ is called the center of $L$; its elements are called central. The fundamental result on commutativity is the following.

Presented to the Society, January 27, 1977; received by the editors April 27, 1976 and, in revised form, April 18, 1977.

AMS (MOS) subject classifications (1970). Primary 06A35, 06A25; Secondary 46C05, 46L10, 81A12.

Key words and phrases. Distributive sublattice, projection lattice, quantum mechanics. 
THEOREM (Foulis-Holland). If any one of $a, b$ or $c$ commutes with the other two, then $(x \vee y) \wedge z=(x \wedge z) \vee(y \wedge z)$ and $(x \wedge y) \vee z=(x \vee z)$ $\wedge(y \vee z)$ where $\{x, y, z\}=\{a, b, c\}$.

The results cited above are lucidly explained in [1, pp. 167-182].

The following result was proved by E. Marsden [6] in 1972 using complicated formulae involving commutators. The proof was considerably simplified in 1973 by L. Herman [7]. The result has not appeared in the literature and I wish to thank both authors for permission to include a proof of it here.

LemMa (MARSDEn-Herman). If any two of $a, b, c$ or $d$ commute with the other two, then

$$
(w \vee x) \wedge(y \vee z)=(w \wedge y) \vee(w \wedge z) \vee(x \wedge y) \vee(x \wedge z)
$$

and

$$
(w \wedge x) \vee(y \wedge z)=(w \vee y) \wedge(w \vee z) \wedge(x \vee y) \wedge(x \vee z)
$$

where $\{w, x, y, z\}=\{a, b, c, d\}$.

The proof of this lemma relies on two remarks. The first of these is an easy application of the Foulis-Holland Theorem; the second is a result of $\mathbf{S}$. S. Holland, Jr. [5, Lemma 7, p. 336].

ReMARK 1. If at most one pair of $a, b, c$ or $d$ fail to commute with each other, then $(a \bigvee b) \wedge(c \vee d)=(a \wedge c) \vee(a \wedge d) \vee(b \wedge c) \vee(b \wedge d)$

Remark 2. If $\{a, c\} \perp\{b, d\}$, then $(a \oplus b) \wedge(c \oplus d)=(a \wedge c) \oplus(b \wedge$ d).

Proof of Lemma. The second statement clearly follows from the first by taking orthocomplements. Moreover, it is a simple computation, using the Foulis-Holland Theorem, that $\{a, b\} \mathbf{C}\{c, d\}$ implies $(a \vee b) \wedge(c \vee d)=$ $(a \wedge c) \vee(a \wedge d) \vee(b \wedge c) \vee(b \wedge d)$. Thus it only remains to show that $\{a, c\} \mathbf{C}\{b, d\}$ implies $(a \vee b) \wedge(c \vee d)=(a \wedge c) \vee(a \wedge d) \vee(b \wedge c)$ $\vee(b \wedge d)$.

Here the right side of the equation is clearly less than the left side. It suffices to show that $(a \vee b) \wedge(c \vee d)-(a \wedge d) \vee(b \wedge c) \leqslant(a \wedge c) \vee$ $(b \wedge d)$, for then $(a \vee b) \wedge(c \vee d) \leqslant(a \wedge c) \vee(a \wedge d) \vee(b \wedge c) \vee(b \wedge$ d) as well.

We proceed to compute

$$
\begin{aligned}
e & =(a \vee b) \wedge(c \vee d)-(a \wedge d) \vee(b \wedge c) \\
& =(a \vee b) \wedge(c \vee d) \wedge[(a \wedge d) \vee(b \wedge c)]^{\prime} \\
& =(a \vee b) \wedge(c \vee d) \wedge(a \wedge d)^{\prime} \wedge(b \wedge c)^{\prime} \\
& =(a \vee b) \wedge(c \vee d) \wedge\left(a^{\prime} \vee d^{\prime}\right) \wedge\left(b^{\prime} \vee c^{\prime}\right) \\
& =\left[(a \vee b) \wedge\left(a^{\prime} \vee d^{\prime}\right)\right] \wedge\left[(c \vee d) \wedge\left(b^{\prime} \vee c^{\prime}\right)\right]
\end{aligned}
$$

Now as $\left\{a, a^{\prime}\right\} \mathbf{C}\left\{b, a^{\prime}, d\right\}$, we have $(a \vee b) \wedge\left(a^{\prime} \vee d^{\prime}\right)=\left(a \wedge d^{\prime}\right) \vee(b$ $\left.\wedge a^{\prime}\right) \vee\left(b \wedge d^{\prime}\right)$ by Remark 1; similarly $(c \vee d) \wedge\left(b^{\prime} \vee c^{\prime}\right)=\left(c \wedge b^{\prime}\right) \vee$ $\left(d \wedge b^{\prime}\right) \vee\left(d \wedge c^{\prime}\right)$. Hence 


$$
\begin{aligned}
e= & {\left[\left(a \wedge d^{\prime}\right) \vee\left(b \wedge a^{\prime}\right) \vee\left(b \wedge d^{\prime}\right)\right] \wedge\left[\left(c \wedge b^{\prime}\right) \vee\left(d \wedge b^{\prime}\right) \vee\left(d \wedge c^{\prime}\right)\right] } \\
= & \left\{\left[\left(a \wedge d^{\prime}\right) \oplus\left(b \wedge a^{\prime}\right)\right] \vee\left(b \wedge d^{\prime}\right)\right\} \\
& \wedge\left\{\left[\left(c \wedge b^{\prime}\right) \oplus\left(d \wedge c^{\prime}\right)\right] \vee\left(d \wedge b^{\prime}\right)\right\} .
\end{aligned}
$$

By hypothesis, any polynomial in $b$ and $d$ (and in $a$ and $c$ as well) is in the centralizer of $\{b, d\}$ if and only if it is in the centralizer of $\{a, b, c, d\}$; so for example, $b \wedge d^{\prime}$ and $d \wedge b^{\prime}$ are in the centralizer of $\{a, b, c, d\}$. Thus by Remark 1 again,

$$
e=\left[\left(a \wedge d^{\prime}\right) \oplus\left(b \wedge a^{\prime}\right)\right] \wedge\left[\left(c \wedge b^{\prime}\right) \oplus\left(d \wedge c^{\prime}\right)\right],
$$

as the other three meets are 0 . But $\left\{a \wedge d^{\prime}, c \wedge b^{\prime}\right\} \perp\left\{b \wedge a^{\prime}, d \wedge c^{\prime}\right\}$; so by Remark $2, e=\left[\left(a \wedge d^{\prime}\right) \wedge\left(c \wedge b^{\prime}\right)\right] \oplus\left[\left(b \wedge a^{\prime}\right) \wedge\left(d \wedge c^{\prime}\right)\right] \leqslant(a \wedge c) \vee$ $(b \wedge d)$. This completes the proof.

Following Crown [2] we call a nonempty set $S \subseteq L$ a Foulis-Holland set provided that whenever $a, b, c$ are distinct elements of $S$ at least one of $a, b, c$ commutes with the other two. The four element set in the hypothesis of the Marsden-Herman Lemma forms a Foulis-Holland set. Crown [2] proved that if $S$ is a Foulis-Holland set which is contained in a modular sublattice of $L$ then the sublattice generated by $S$ is distributive. In the next section we shall prove this result without the modularity hypothesis, thereby answering the question posed by Crown [2].

2. The Main Theorem. For $N \subseteq L$, let $\langle N\rangle$ denote the sublattice of $L$ generated by $N$ and, for $x \in L$, let $x \wedge N=N \wedge x$ denote the set $\{x \wedge n \mid n$ $\in N\}$. Note that $x^{\prime}$ need not be in $\langle\{x\}\rangle$. Let $\mathscr{P}(S)$ denote the power set of the set $S$ and ${ }^{\#} S$ the cardinality of $S$.

REMARK 2.1. Let $e \in L$ and $D, N, S \subseteq L$.

(1) If $S$ is a finite Foulis-Holland set with an odd number of elements, then $S \cap C(S) \neq \varnothing$.

(2) If $D$ is a distributive sublattice of $L$ and $e \in C(D)$ then $\langle D \cup\{e\}\rangle$ is distributive.

(3) If $e \in C(N)$ and $\bigvee N$ as well as $\bigvee(N \wedge e)$ exist in $L$ then $e \wedge(\bigvee N)$ $=\bigvee(e \wedge N)$.

Let $e_{1}, e_{2}, \ldots, e_{n}$ be a maximal orthogonal set of nonzero central elements of $L$. Then

(4) $L$ is ortho-isomorphic to $\prod_{i=1}^{n} L\left[0, e_{i}\right]$, and

(5) $\langle S\rangle$ is isomorphic to a sublattice of $\prod_{i=1}^{n}\left\langle S \wedge e_{i}\right\rangle$.

Proof. Parts (1), (4) and (5) lend themselves to easy induction arguments. The first part hinges on the fact that if $S$ is a Foulis-Holland set and $s \in S \backslash C(S)$ then there exists a unique $t \in S \backslash C(S)$ such that $s \in C(S \backslash$ $\{t\})$ and $t \in C(S \backslash\{s\}) ; S \backslash\{s, t\}$ is again a Foulis-Holland set. Part (2) was first observed by Crown: note that $e$ is neutral in $\langle D \cup\{e\}\rangle$ by the FoulisHolland Theorem; thus, if $D_{1}$ is a maximal distributive sublattice of $\langle D \cup$ $\{e\}\rangle$ containing $D$ then $e \in D_{1}$ so that $D_{1}=\langle D \cup\{e\}\rangle$ is distributive. A proof of part (3) may be found in [4, Theorem 7]. 
LEMMA 2.2. Let $S=\left\{s_{1}, \ldots, s_{n}, t_{1}, \ldots, t_{n}\right\}$ be a finite nonempty subset of $L$, where $s_{i} \in C\left(S \backslash\left\{t_{i}\right\}\right)$ and $t_{i} \in C\left(S \backslash\left\{s_{i}\right\}\right)$, for $i=1, \ldots, n$, and let $A_{S}=\left\{x_{1} \wedge \cdots \wedge x_{n} \mid x_{i} \in\left\{s_{i}, t_{i}\right\}\right\} \backslash\{0\}$. If $s_{i}$ and $t_{i}$ are complements in $L$, for $i=1,2, \ldots, n$, then $\mathcal{P}\left(A_{S}\right) \simeq\langle S\rangle$.

Proof. Define $\psi: \mathcal{P}\left(A_{S}\right) \rightarrow\langle S\rangle$ by the rule $\psi(M)=\bigvee M$ for $M \subseteq A_{S}$. For $M \subseteq A$, define $\delta(M)=\left\{x_{2} \wedge \cdots \wedge x_{n} \mid\right.$ for some $x_{i} \in\left\{s_{1}, t_{1}\right\}, x_{1}$ $\left.\wedge \cdots \wedge x_{n} \in M\right\}$ and, for $y_{1} \in\left\{s_{1}, t_{1}\right\}, M_{y_{1}}=\left\{\bigwedge_{i=1}^{n} x_{i} \in M \mid y_{1}=x_{1}\right\}$.

Claim. For $\{m\} \cup N \subseteq A_{S}$,

$$
m \wedge \bigvee N= \begin{cases}m & \text { if } m \in N, \\ 0 & \text { if } m \notin N .\end{cases}
$$

We prove the claim by induction on $n$, the case $n=1$ being trivial. We may assume that $m=s_{1} \wedge x_{2} \wedge \cdots \wedge x_{n}$ so that $m \in N$ if and only if $\delta m \in$ $\delta\left(N_{s_{1}}\right)$. Now

$$
\begin{aligned}
m & \wedge(\vee N)=s_{1} \wedge \delta m \wedge\left(\bigvee \delta N_{s_{1}} \vee \bigvee \delta N_{t_{1}}\right) \\
& =s_{1} \wedge \delta m \wedge\left[\left(s_{1} \wedge \bigvee \delta N_{s_{1}}\right) \vee\left(t_{1} \wedge \bigvee \delta N_{t_{1}}\right)\right] \\
& =s_{1} \wedge \delta m \wedge\left(s_{1} \vee\left(\bigvee \delta N_{t_{1}}\right)\right) \wedge\left(t_{1} \vee \bigvee \delta N_{s_{1}}\right) \wedge\left(\bigvee \delta N_{s_{1}} \vee \vee \delta N_{t_{1}}\right)
\end{aligned}
$$

by the Marsden-Herman Lemma and the fact that $s_{1} \vee t_{1}=1$. Thus

$$
\begin{aligned}
m \wedge \bigvee N= & \delta m \wedge s_{1} \wedge\left(t_{1} \vee \bigvee \delta N_{s_{1}}\right) \wedge(\bigvee \delta N) \\
= & \delta m \wedge s_{1} \wedge \vee \delta N_{s_{1}} \wedge \vee \delta N \\
& \text { by the Foulis-Holland Theorem and the fact that } s_{1} \wedge t_{1}=0 \\
= & s_{1} \wedge \delta m \wedge \vee \delta N_{s_{1}} \\
= & \left\{\begin{array}{ll}
s_{1} \wedge \delta m & \text { if } \delta m \in \delta N_{s_{1}}, \\
s_{1} \wedge 0 & \text { if } \delta m \notin \delta N_{s_{1}},
\end{array}=\right. \\
= & \begin{cases}m & \text { if } m \in N, \\
0 & \text { if } m \notin N .\end{cases}
\end{aligned}
$$

We have invoked the induction in the second last step and the claim is proved. It follows immediately that $M \subseteq N$ if and only if $\psi(M) \leqslant \psi(N)$. Thus, in order to prove that $\psi$ is an isomorphism, we need only prove that it is onto. This reduces to proving that $S \subseteq \operatorname{image}(\psi)$ and, by symmetry, that $s_{1} \in$ image $(\psi)$. This is trivial for $n=1$; and, if we assume that it is true for all $1<k<n$, then $s_{2}=\bigvee F$ and $t_{2}=\bigvee G$ for some $F, G \subseteq \delta\left(A_{S}\right)$. Thus

$$
\begin{aligned}
s_{1} & =s_{1} \wedge\left(s_{2} \vee t_{2}\right)=s_{1} \wedge((\bigvee F) \vee(\bigvee G)) \\
& =\left(s_{1} \wedge(\bigvee F)\right) \vee\left(s_{1} \wedge(\bigvee G)\right)=\bigvee\left(s_{1} \wedge F\right) \bigvee \bigvee\left(s_{1} \wedge G\right)
\end{aligned}
$$

by the Foulis-Holland Theorem and Remark 2.1, part (3). Hence $s_{1}$ is the join of elements from image $(\psi)$ and $\psi$ is onto. It follows that $\psi$ is a lattice isomorphism from $\mathscr{P}\left(A_{S}\right)$ onto $\langle S\rangle$. The lemma is proved. 
MAIN THEOREM. If $S$ is a Foulis-Holland subset of the orthomodular lattice $L$, then $\langle S\rangle$ is distributive.

Proof. If $p, q, r \in\langle S\rangle$ then there exists a finite set $T \subseteq S$ such that $p, q$, $r \in\langle T\rangle \subseteq\langle S\rangle$. Thus the verification of distributivity in $\langle S\rangle$ reduces to its verification in such $\langle T\rangle$. We therefore may assume that $S$ is a finite set. For convenience of notation we shall assume that the sub-ortholattice $[S]$ generated by $S$ is $L$. The proof is by induction on ${ }^{\#}(S)$. The result is trivial for ${ }^{\#} S=1$. Let ${ }^{\#} S=k>1$ and assume that all Foulis-Holland sets of cardinality less than $k$ generate distributive sublattices. By Remark 2.1 parts (1) and (2) we may assume that $k=2 n$ and that $S=$ $\left\{s_{1}, s_{2}, \ldots, s_{n}, t_{1}, t_{2}, \ldots, t_{n}\right\}$ where $s_{i} \in C\left(S \backslash\left\{t_{i}\right\}\right)$ and $t_{i} \in C\left(S \backslash\left\{s_{i}\right\}\right)$, for $i=1,2, \ldots, n$.

Let $E=\left\{e_{1}, e_{2}, \ldots, e_{2 n+1}\right\}$ where, for $1 \leqslant i \leqslant n$,

$$
\begin{aligned}
e_{i} & =\left(s_{i} \vee t_{i}\right)^{\prime} \wedge \bigwedge_{j<i}\left(s_{j} \vee t_{j}\right), \\
e_{n+i} & =\bigwedge_{j<i}\left(\left(s_{j} \vee t_{j}\right) \wedge\left(s_{j} \wedge t_{j}\right)^{\prime}\right) \wedge\left(s_{i} \wedge t_{i}\right) \wedge \bigwedge_{j>i}\left(s_{j} \vee t_{j}\right)
\end{aligned}
$$

and

$$
e_{2 n+1}=\bigwedge_{i=1}^{n}\left(s_{i} \vee t_{i}\right) \wedge\left(s_{i} \wedge t_{i}\right)^{\prime}
$$

A simple induction argument proves that $E \cup\{0\}$ is a maximal set of orthogonal central elements of $[S]=L$, so that $L$ is ortho-isomorphic to $\Pi_{e_{i} \in E \backslash\{0\}} L\left[0, e_{i}\right]$. Let $S_{i}=S \wedge e_{i}$ and note that $S_{i}$ is a Foulis-Holland set for all $i$. Moreover, for $i \leqslant 2 n,{ }^{\#}\left(S_{i} \backslash\{0\}\right)<^{\#}(S)$; to see this, define $\phi_{i}: S \rightarrow S_{i}$ from $S$ onto $S_{i}$ by $\phi_{i}(S)=S \wedge e_{i}$ and observe that if $i \leqslant n$ then $\phi_{i}\left(s_{i}\right)=$ $\phi_{i}\left(t_{i}\right)=0$ and $\phi_{n+i}\left(s_{i}\right)=\phi_{n+i}\left(t_{i}\right)=e_{n+i}$ so that no $\phi_{i}$ is an injection. It follows by induction that, for $i \leqslant 2 n,\left\langle S_{i}\right\rangle$ is distributive. Now, in $L\left[0, e_{2 n+1}\right]$, the generating elements satisfy

$$
\left(s_{i} \wedge e_{2 n+1}\right) \vee\left(t_{i} \wedge e_{2 n+1}\right)=e_{2 n+1}
$$

and

$$
\left(s_{i} \wedge e_{2 n+1}\right) \wedge\left(t_{i} \wedge e_{2 n+1}\right)=0
$$

so that, by Lemma $2.2,\left\langle S_{2 n+1}\right\rangle$ is distributive. Finally, by Remark 2.1 part (5), $\langle S\rangle$ is isomorphic to a sublattice of $\prod_{i=1}^{2 n+1}\left\langle S_{i}\right\rangle$ which is distributive. The theorem is proved.

We conclude by noting that there exist simple examples showing that a distributive sublattice of an orthomodular lattice need not be generated by a Foulis-Holland set.

\section{REFERENCES}

1. T. S. Blyth and M. F. Janowitz, Residuation theory, Pergamon Press, Oxford, 1972.

2. G. Crown, A note on distributive sublattices of an orthomodular lattice, J. Natur. Sci. and Math. 16 (1976), 75-80.

3. D. J. Foulis, A note on orthomodular lattices, Portugal. Math. 21 (1962), 65-72. 
4. S. S. Holland, Jr., A Radon-Nikodym theorem in dimension lattices, Trans. Amer. Math. Soc. 108 (1963), 66-87.

5. Distributivity and perspectivity in orthomodular lattices, Trans. Amer. Math. Soc. 112 (1964), 330-343.

6. E. L. Marsden, A condition for distribution in orthomodular lattices, Kansas State Univ. Technical Report \#23, 1972.

7. E. L. Marsden and L. M. Herman, A condition for distribution in orthomodular lattices, Kansas State Univ. Technical Report \#40, 1974.

Department of Mathematics, Kansas State University, Manhattan, Kansas 66506 